\title{
A High-duty 1.6 Cell s-Band RF Gun Driven By a psec Nd:YAG Laser"
}

\author{
Y. Aoki", J. Yang, M. Yorozu, Y. Okada, A. Endo /SHI, Yato, Tanashi, Japan/ \\ T. Kozawa, Y. Yoshida, S. Tagawa /ISIR, Osaka Univ., Mihogaoka, Ibaragi, Japan/ \\ M. Washio /RISE, Waseda Univ., Ohkubo, Shin-juku, Japan / \\ X.Wang, I. Ben-Zvi /ATF, BNL, Upton, NY/
}

\begin{abstract}
The performance tests were done for a 1.6 cell s-band BNL-type photocathode RF electron gun, GUN-IV, under a condition of $1.5 \mathrm{MW}$ RF power input and $266 \mathrm{~nm}$ Nd:YAG laser pulse irradiation. As a result, the maximum energy and the maximum charge/ bunch were obtained as $1.6 \mathrm{MeV}$ and $120 \mathrm{pC} /$ bunch, respectively. In addition, a sinusoidal behavior of current with the polarization angle of laser light were measured. Furthermore, the change in current as a function of laser injection phase was measured and reasonably reproduced by a numerical calculation.
\end{abstract}

\section{INTRODUCTION}

Recently, photocathode RF electron guns are being vigorously developed by several groups for free electron laser, laser- and plasma- accelerations, laser Compton scattering experiments etc., since this kind of gun has several advantages. First, ultra-low emittance beam can be realised. And then, any bunching section is not necessary in an accelerator, energy spread can be largely suppressed, bunch length can be controlled by the pulse width of laser, the synchronization can be easily achieved with laser pulse, and so on.

A new BNL-type photocathode RF gun (GUN IV) was designed and constructed under the BNL/KEK/SHI international collaboration in 1997[1], based on the design of BNL-GUN III[2]. Here, the second generation of the GUN IV is introduced, which was manufactured by SHI for ultra-short X-ray pulse generation through the inverse Compton scattering $[3,4]$.

In this paper, some results from the performance tests of this gun, such as the electron energy and the dependence of the beam characteristics on the parameter of laser injection, are shown. Though the designed peak power of RF pulse is $7 \mathrm{MW}$, only $1.5 \mathrm{MW}$ power was fed into the gun due to the limitation of experimental setup. However, the results show the gun can be operated satisfactorily by such low RF power.

"This work was partially performed under the management of the Femtosecond Technology Research Association (FESTA) supported by the New Energy and Industrial Technology Development Organization Japan.

${ }^{\#}$ Email: yss_aoki@shi.co.jp

\section{RF GUN SYSTEM}

Our gun system has almost the same structure as the first generation[1] and can be separated into a gun cavity (1.6 cell), a single emittance compensation solenoid magnet[5], a pair of dipole magnet and a vacuum pumping unit. The gun cavity has two s-band cells made of oxygen-free highly pure copper (class 1 ), called "half cell" $(0.6$ cell $)$ and full cell $(1.0$ cell $)$. These cells are directly-coupled. The half cell ended with the photocathode and have two optical ports for laser injection whose angle is $67.5^{\circ}$ to the normal of the photocathode. On the other hand, the full cell has the beam exit and is connected with the waveguide for RF power input. Furthermore, this gun has three water cooling channels for the higher repetition rate in operation (high-duty).

The solenoid magnet is ca. $230 \mathrm{~mm}$ long including yoke plates and directly connected with the gun at oneside. The magnetic field along the central axis of the solenoid coil was measured to be homogeneous over 100 $\mathrm{mm}$ in z-direction and at least $30 \mathrm{~mm}$ in the radial direction. The maximum field strength was $3 \mathrm{kG}$ at 200A.

The dipole magnets for $\mathrm{x}$ - and $\mathrm{y}$ - direction are located in the hole of the solenoid magnet and have the common central axis in z-direction with the solenoid. The straight section of the coil is $100 \mathrm{~mm}$ long and can give homogeneous field. The field strength shows a linear relationship with the current and $40 \mathrm{G}$ was measured at the current of $1 \mathrm{~A}$.

The gun cavity was directly pumped out by an ion pump $(150 \mathrm{l} / \mathrm{s})$ and the base pressure was achieved to about $1 \times 10^{-6} \mathrm{~Pa}$. An auxiliary turbo molecular pump system is set in the down stream of the solenoid coil. It is used only for starting up evacuation of the system.

\section{ELECTRON BEAM GENERATION}

The performance tests were done in the LINAC facility of ISIR, Osaka University. The power of input RF was measured through a directional coupler to be $1.5 \mathrm{MW}$. The laser which drives the gun was a 15 picosecond $\mathrm{Nd}$ :YAG laser which is developed especially for RF guns by Time Bandwidth Products (TBP) and Sumitomo Heavy Industries (SHI)[6].

The profile of electron beam was monitored by the combination of a phosphor plate, which is made of sintered $\mathrm{Al}_{2} \mathrm{O}_{3}$ doped with $\mathrm{Cr}$, and a $\mathrm{CCD}$ camera (screen 
monitor). Fig. 1 shows the optimal beam profile in the form of the cross sectional views along $x$ axis(left) and $y$ axis(right) obtained after the solenoid. The fitting of the profile with a gaussian curve resulted in the beam size of about $1.5 \mathrm{~mm} \phi\left(2 \sigma_{\mathrm{x}}=1.48 \mathrm{~mm}\right.$ and $\left.2 \sigma_{\mathrm{y}}=1.53 \mathrm{~mm}\right)$.
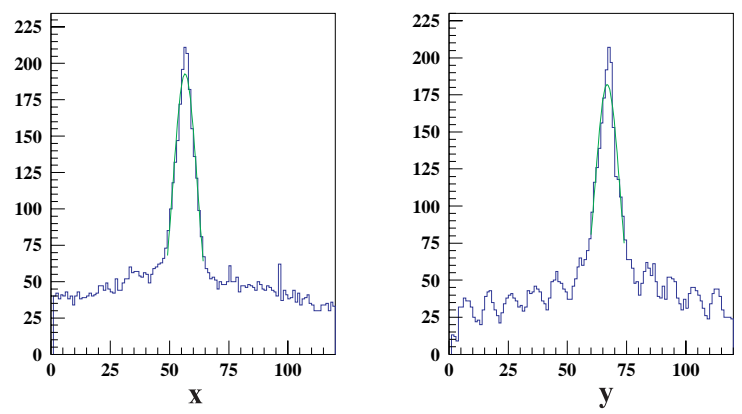

Fig. 1 The cross-sectional view of the optimal beam profile (x-axis:left, $y$-axis:right), observed on the screen monitor after the solenoid magnet. The dimension is described in unit of pixels (1pixel $=0.287 \mathrm{~mm}$ ). The oscillation at low level in the right figure might be some back-ground frequency noise on measurement.

\section{ENERGY MEASUREMENT}

The energy of electron beam was measured by the steering action of the magnetic fields on the beam. In the measurement, the dipole(x) field was set and fixed to the appropriate value and the distance of the center in the beam profile from the original one $\left(B_{\text {dipole }(x)}=0\right)$ was measured. Then, changing the solenoid field, the distance was measured. The results are seen in Fig. 2 with three simulation curves. The beam trajectory was calculated based on a simple Lorenzian eq. in the simulation for the electron beam energy of $1.5-1.7 \mathrm{MeV}$.

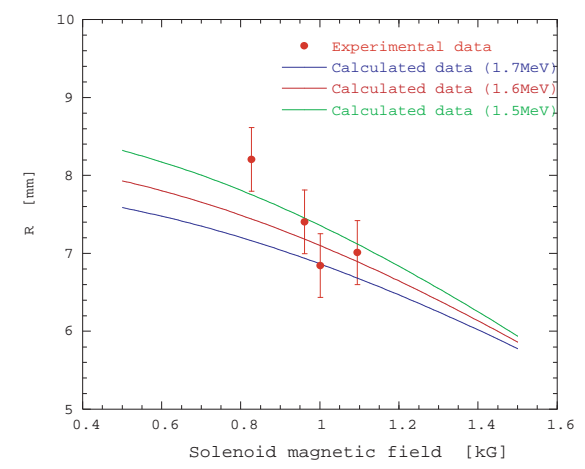

Fig. 2 The radial distance of the beam position from the original one, observed on the screen monitor. The solenoid field was changed while the dipole field was fixed. The simulation curves are for $1.5,1.6$ and $1.7 \mathrm{MeV}$ electron beams.

From this figure, in the case of $1.5 \mathrm{MW}$ power input, approximately $1.6 \mathrm{MeV}$ of beam energy was obtained.
At this energy, emission intensity was not so sufficient in the measurement then the data are relatively scattered. The error bars in Fig. 2 show the resolution of the CCD camera (1 pixel).

On the other hand, the maximum acceleration field in the gun is calculated to $17 \mathrm{MV} / \mathrm{m}$, using the RF power of $1.5 \mathrm{MW}$. This acceleration field can give the energy of about $1.9 \mathrm{MeV}$ which is not so different from the experimentally estimated above.

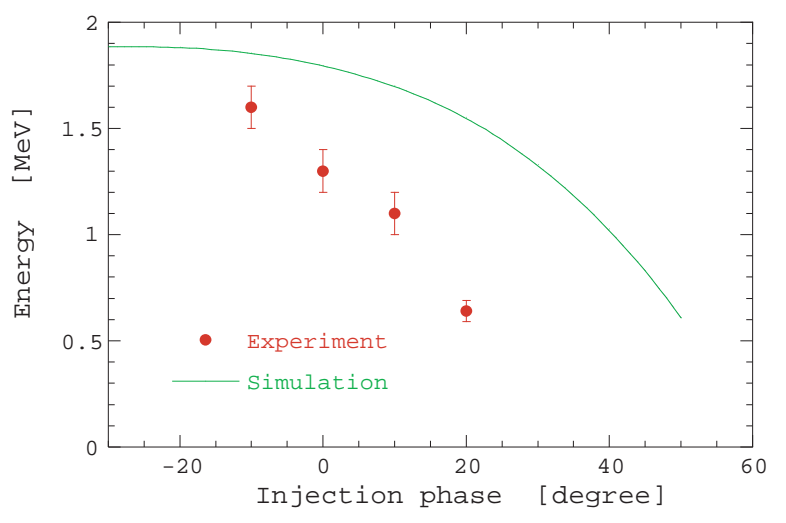

Fig. 3 The dependence of the electron energy on the laser injection phase. Error bars indicate the resolution of the CCD camera.

The dependence of the energy on laser injection phase was also measured. The trend of change in energy is similar to the curve from simulation which was done with the calculated acceleration field. The curve indicates that acceleration efficiency become lower by the phase change. The difference between the measured values and the simulated curve seems enhanced with increase in phase. It may come from scattering effects by residual gas, because the RF conditioning is not sufficient yet.

\section{CHARGE MEASUREMENT}

Current of the output beam was measured with a thick copper electrode $(10 \mathrm{~mm} \phi)$ with impedance-matched to 50 ohm which is located at $730 \mathrm{~mm}$ from the photocathode. The charge of the bunch was estimated from the current, considering the repetition rate of $10 \mathrm{~Hz}$ and subtracting the dark current.

In Fig. 4, the measured charge is plotted as a function of laser injection phase. The simulated curve which is normalized at the maximum point is also shown in the figure. The maximum current was obtained at the phase somewhat before that giving the maximum field (phase $=0)$. It is the same situation as in the case of energy, seen in Fig. 3. Over 30 degree in Fig. 4, a small peak appears. This may be because the RF power of $1.5 \mathrm{MW}$ was used instead of the designed power of $7 \mathrm{MW}$. It is suspected 
that electron beam might have a complicated trajectory between the half cell and full cell.

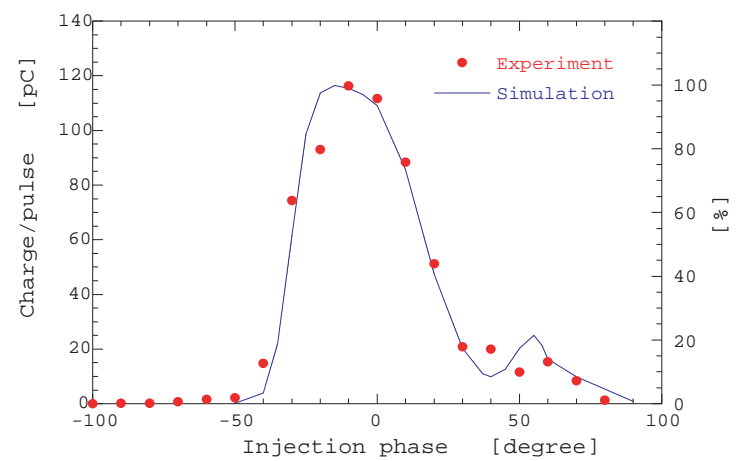

Fig. 4 The charge measurement as a function of the laser injection phase and the simulation cueve.

The effect of polarization angle of laser light on the charge was measured by using a $1 / \lambda$ plate. As shown in Fig. 5, sinusoidal change in charge was obtained. As Sakai et al. pointed out[1], since we inject laser pulse onto the photocathode, one directional component of the field of laser light may affect much largely on the photoemission process at the cathode. The maximum effect should be obtained at p-type polarization.

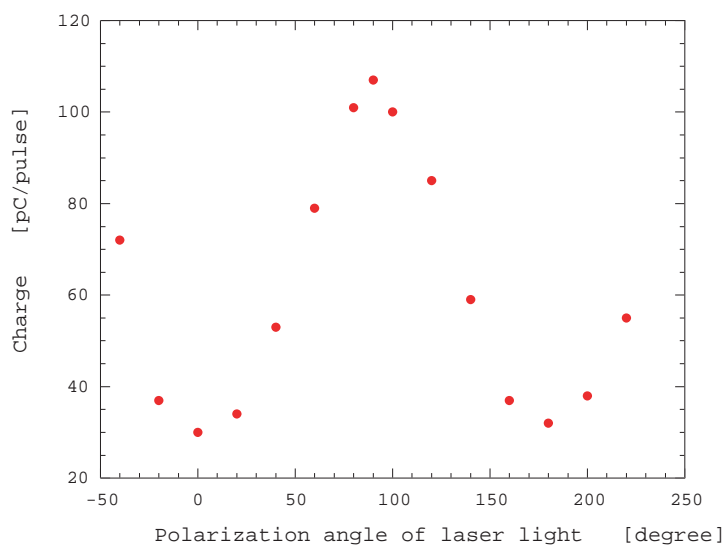

Fig. 5 The change in charge/pulse with the laser injection phase.

After a series of performance tests described above, we have got the optimum parameters for the solenoid field and for the laser injection to get the maximum electron charge from the gun. Finally, the maximum charge was recorded $120 \mathrm{pC} /$ pulse at the solenoid field of $0.6 \mathrm{kG}$ and the laser injection phase of -10 degree, while the dark current was $60 \mathrm{pC} / \mathrm{RF}$ pulse. As discussed on Fig. 4, the insufficient RF conditioning can make the gun performance worse. The relatively high dark current may come from this condition. In future, using high power RF, the performance test including the emittance measurement and the bunch length measurement will be done and our project on ultra-short X-ray generation through the inverse Compton scattering will be proceeded.

\section{CONCLUSION}

The performance test of the second generation of GUNIV was done with RF power of $15 \mathrm{MW}$ and $\mathrm{Nd}$ :YAG laser. The maximum energy and the maximum charge/pulse were $1.6 \mathrm{MeV}$ and $120 \mathrm{pC} / \mathrm{pulse}$, respectively. One of the most important advantage of RF gun is ultra-low emittance beam generation based on the large acceleration gradient $(>100 \mathrm{MV} / \mathrm{m})$ induced by high power RF. Next step, we should perform this kind of experiment. But here, in other words, we demonstrated that the gun works well even with such low power RF (1.5MW).

\section{ACKNOWLEDGEMENTS}

The authors would like to thank Mr. Yamamoto and Mr. Saeki for their helpful, technical support in the performance tests.

\section{REFERENCES}

[1] F. Sakai et al., The proc. 11th Symp. Acc. Sci. Tech., Harima, pp.473-475 (1997).

[2] X. Wang et al., The proc. PAC '93 pp.3000-3002 (1993).

[3] M. Washio et al., The proc. 7th Chine-Japan Bilateral Symp. Radiat. Chem., Chengdu, China (1996).

[4] J. Yang et al, The proc. 6th EPAC, Stockholm, pp.1082-1084 (1998).

[5] D.T. Palmer et al., The proc. PAC '97, Vancouver, pp.2843-2845 (1998).

[6] F. Sakai et al., PAC '99, New York (1999). 